\title{
Analyzing Interdependent National Infrastructure Provisions Under Extreme Climate Risk
}

\author{
Raghav Pant ${ }^{\mathrm{a}}$ \\ Scott Thacker ${ }^{\mathrm{a}}$ \\ Jim W. Hall ${ }^{\mathrm{a}}$ \\ Stuart Barr ${ }^{\mathrm{b}}$ \\ David Alderson ${ }^{\mathrm{b}}$
}

\begin{abstract}
To create sustainable, adaptive and resilient societies we need to develop a proper understanding of infrastructure risk. This research improves such understanding by examining widespread failures of interdependent infrastructure networks from extreme climate events. By subjecting infrastructure networks to extreme climate loading, we construct ensembles of direct failure sets that lead to cascading indirect failures across topological infrastructure networks. Such analysis produces two results: (1) Estimations of the topological fragility of infrastructure networks, and (2) Infrastructure vulnerability quantification in terms of spatial affects on service provision and customers using networked infrastructures. Producing multiple failure sets provides a wide range of possible outcomes, helping to build infrastructure failure profiles. Insights from risk analysis strengthen our understanding of infrastructure failures and are used to inform resilience-building activities for effective infrastructure provision.
\end{abstract}

\section{Key words: Infrastructure risk; Interdependence; Climate hazards}

\section{Introduction}

Infrastructure sustainability, adaptation or resilience is better understood by examining national infrastructure risk. In the context of this research, national infrastructures represent civil and technological structures that provide goods and services to industries, governments and households operating at regional and national scales. In particular infrastructures such as electricity, gas, rail, road, and ICT are among critical national infrastructures, constituting the backbone of society and economy ${ }^{1,2}$. For better performance and service provision national infrastructures are highly interdependent systems through physical, technological or economic mechanisms ${ }^{3}$.Though interdependencies are desirable for maintaining infrastructure functionality and service delivery, they become disadvantageous during widespread failures, which result in failure cascading effects that propagate damages from one infrastructure to another ${ }^{4}$.

${ }^{a}$ Environmental Change Institute, University of Oxford, South Parks Road, Oxford OX1 3QY, UK, jim.hall@eci.ox.ac.uk

${ }^{\mathrm{b}}$ School of Civil Engineering and Geosciences, Newcastle University, Newcastle Upon Tyne NE1 7RU, UK.

http://dx.doi.org/10.14453/isngi2013.proc.35 
Extreme climate events put national infrastructures at risk because they are capable of causing widespread social and economic losses. Notably extreme hurricanes in the United States, floods in United Kingdom, and extreme heat in Australia have highlighted the large-scale vulnerabilities of national infrastructures provisions ${ }^{5,6,7}$. National scale climate change risk assessment policy statements have emphasized the imminent risks to critical national infrastructures in the present and future ${ }^{8,9}$. Climate risk modeling is inherently complex due to systematic uncertainties that propagate from extreme climate hazards towards infrastructure responses and failure impacts ${ }^{10}$. For critical national infrastructures a system-of-systems (SoS) approach is required for modeling escalating failures that affect multiple systems and multiple participants ${ }^{11}$. In this paper we present a SoS framework where infrastructures represent systems of interdependent spatial networks that are exposed to probabilistic extreme hazard scenarios.

Infrastructures are spatially distributed systems spread over large geographic areas. Further there are several components or assets within each infrastructure and across infrastructures that are connected physically or through flow of information. As such an overall spatial network topology can be identified to build a unified representation of infrastructures ${ }^{12}$. Extreme climate loading conditions initiate random failures of network assets and the topology determines the further propagation of these failures across entire networks ${ }^{13}$. Following network damages the SoS risks are quantified in terms of the consequences in terms of spatial damage impacts, demographic disruption impacts and interdependent economic loss impacts ${ }^{14}$.

The climate risk analysis methodology proposed in this paper aims to compute the overall risk of failure of infrastructure networks when exposed to multiple probabilistic climate hazards. By subjecting infrastructure networks to extreme climate loading, we construct ensembles of direct asset failure sets that lead to cascading indirect failures across topological infrastructure networks. Such analysis produces two results: (i) Estimations of the topological fragility of infrastructure networks, and (ii) Infrastructure vulnerability quantification in terms of spatial affects on service provision and customers using networked infrastructures. Producing multiple failure sets provides a wide range of possible outcomes, helping to build infrastructure failure profiles. Insights from risk analysis strengthen our understanding of infrastructure failures and are used to inform resilience-building activities for effective infrastructure provisions.

In the sections that follow we first explain the formulation for calculating interdependent infrastructure risk for extreme climate hazards. Next we present the underlying SoS framework that needs to be constructed for implementing the different components of the risk calculations. This is followed by a sample case-study demonstration for a national-scale network and hazard.

\section{Quantifying infrastructure risk}

Infrastructure risk is broadly quantified as the product of the probabilities and consequences of network failures conditional upon probabilistic extreme climate hazards ${ }^{10}$. Within the context of this paper, reliability is the measure of the probability of failure, which is studied at the individual assets level and then at the infrastructure network level. For damage assessment the focus lies in estimating the customer losses and infrastructure output degradations, which 
ultimately are converted to economic losses at appropriate individual asset, network or nationalscale levels.

Probabilistic extreme climate loading is quantified in terms of its spatial magnitude vector and joint probability distribution . To calculate risk we first represent an individual infrastructure asset functionality through a state function, such that denotes a 'failed' state and denotes a 'non-failed' state. Also we define two variables: (i) : The conditional probability of failure of an asset (fragility) when subjected to the external hazard loading , (ii) : The damage associated with the failure of the asset. For the entire infrastructure network, consisting of states collected into a binary vector assets, functionality depends on all the asset describing which assets have failed and which have not failed. In particular network reliability, damages and risk depend upon the elements in .

When exposed to the hazard there are many possible failure combinations of assets that result in network failure. In the most exhaustive scenario there are possible failure state combinations, but in reality fewer combinations can capture most of the failures. The vector defined before represents just one of the possible failure states and is defined here as a failure mechanism. We define the vector

to represent the failure mechanism and the tensor as the collection of failure combinations that contribute to overall network failure. The infrastructure network risk ( ) formulation proposed in this paper is based on: (i) estimating the combined asset failure probabilities ( ) and damages ( ) due to multiple failure mechanisms, and (ii) repeating the calculations over multiple hazard loadings . This is shown in Equation (1) below and summarized in Figure 1.

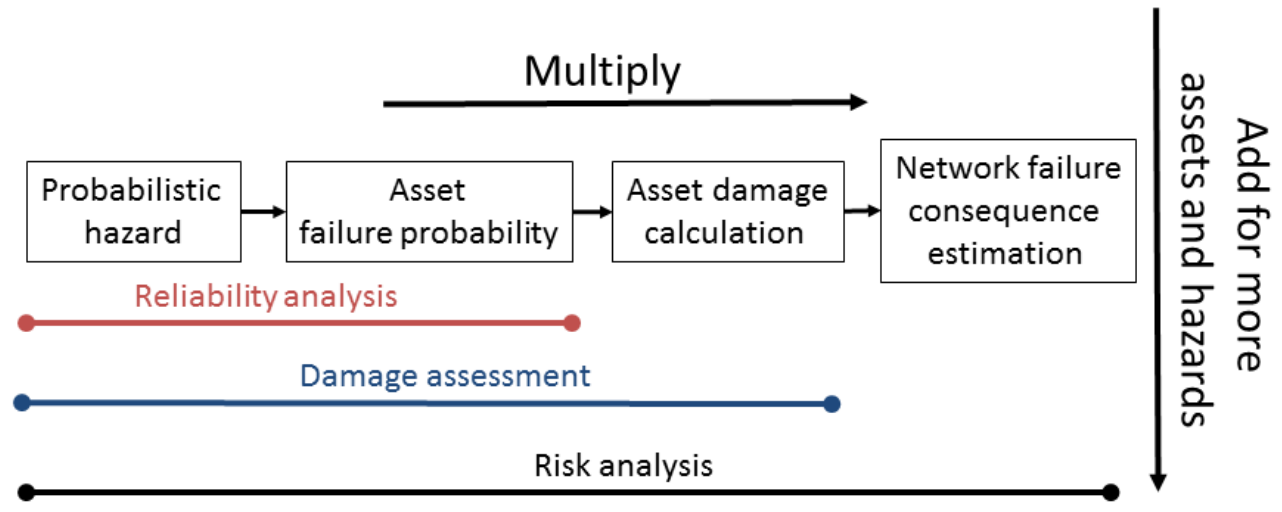

Figure 1. Framework for risk calculations required in the network failure analysis. 


\section{Risk implementation}

Figure 2 shows a detailed flowchart for constructing component models and implementing a national infrastructure risk calculation framework that solves Equation (1). The important components in the flowchart are explained as follows:

1. Hazard estimation (Component A): Different types of extreme climate models can be built to estimate hazard severity (h) and uncertainty $(\mathbf{h})$. Some of the models (Hazard extent maps/Spatial distributions of hazards/Spatial-temporal distributions of hazards) that are used in this framework are shown in the component A.

2. Network estimation (Component B): Topological network representations are essential for generating failures and computing failure probabilities $\left(\mathbb{P}\left[r_{i}^{j} \mid \mathbf{h}\right]\right)$. In this framework networks are built from information on the geo-locations of assets and their physical connectivity to other assets and networks.

3. Network reliability analysis (Component $\mathbf{C}$ ): Reliability analysis provides the framework for building failure mechanisms, which leads to the computation of asset and topological fragilities, and ultimately the network failure probability. In this framework direct failures simulated by Monte-Carlo based approaches and network connectivity are utilised to estimate resulting topological failures.

4. Infrastructure damage assessment (Component D): Damages are quantified spatially by first constructing infrastructure footprints that estimate the number of customers served over the area influence of assets. Following this the direct and indirect spatial and demographic impact effects for asset damages and network losses can be quantified by assembling the footprints of all failed assets. For economic analysis purposes, the total network damage effects constitute direct economic losses due to infrastructure asset failures.

5. Economic damage (loss) assessment (Component E): The supply and demand side loss inputs are fed into an economic input-output model. Using the economic input-output analysis to find the disrupted equilibrium state we can generate the indirect losses and total losses $\left(D\left(r_{i}^{j}\right)\right)$ due to the network failures.

6. Risk calculation (Component F): Network risk $R(\overline{\mathbf{r}})$ is computed when the reliability and damage estimations are implemented over multiple failure mechanisms and multiple hazards. 


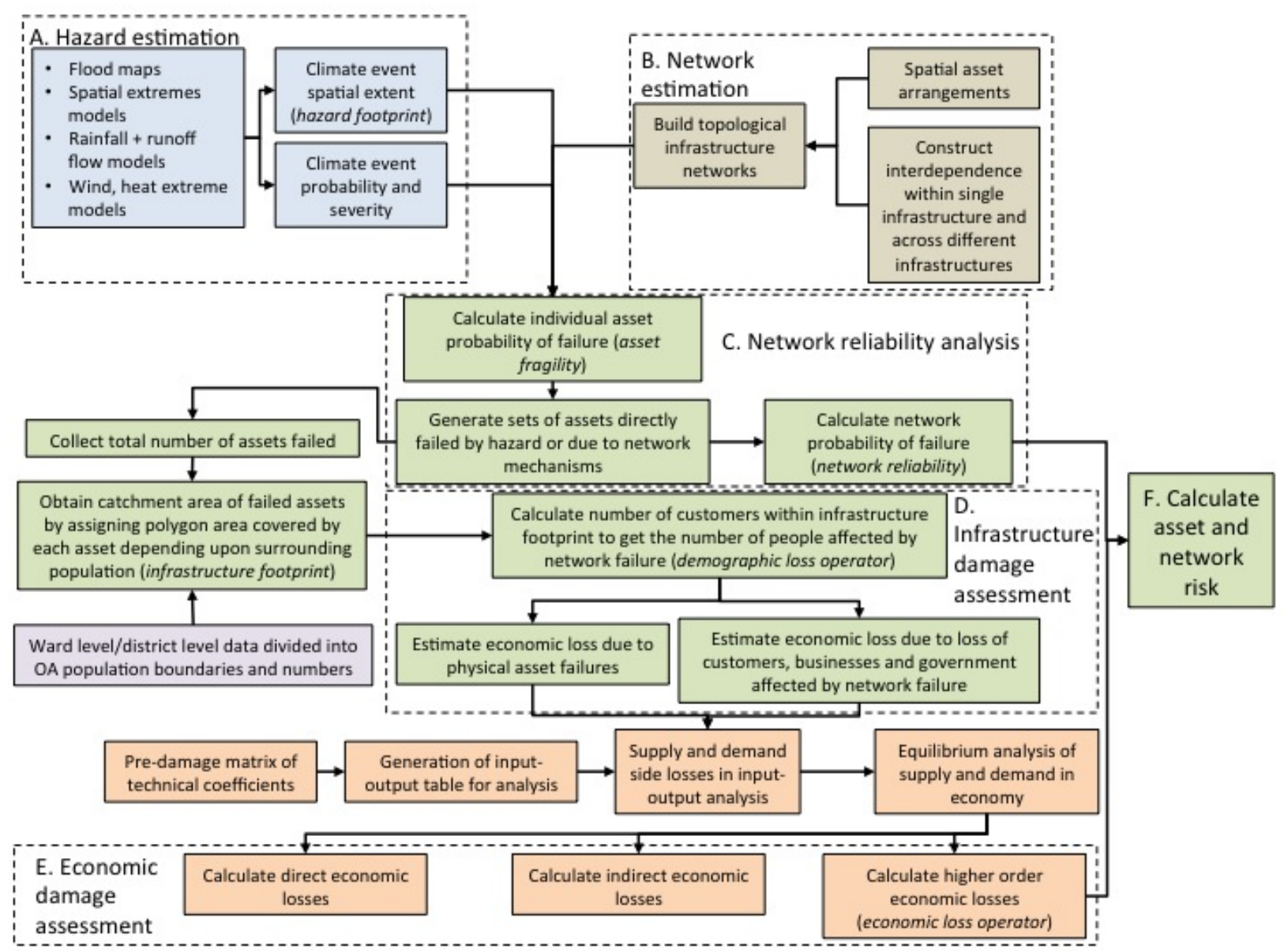

Figure 2: Flowchart showing steps in the ITRC WS2 Phase 2 infrastructure risk analysis.

\section{Case-study demonstration}

Obtaining high quality data for the different components of the risk framework is very challenging, so models are employed wherever necessary. The case-study results shown here are synthetic but serve the important purpose of providing a template for risk calculations when real data is available. The risk methodology outlined in the sections above is implemented for simulated probabilistic hazard events that affect a sample topological electricity network for Great Britain. The network is a satisfactory topological representation of the actual electricity transmission network for Great Britain ${ }^{15}$. Further, the network nodes represent electricity substations that served customers over regions estimated from population census data ${ }^{16}$.

Figure 3(a) introduces the test network with nodes (substation) fragilities, magnified according to their relative values, after being intersected with a sample probabilistic spatial hazard event. This result is obtained by implementing the components $\mathrm{A}, \mathrm{B}$ and $\mathrm{C}$ from the framework Figure 2. Using Figure 3(a) we can identify the substations that are at most risk of failing. Based on the node fragilities and resulting network behaviors we can generate a sample of different possible failure mechanisms. This is shown in Figure 3(b) where the resulting damages in terms of 
customers affected are calculated for each direct and indirect failure mechanism. Hence, we are also able to obtain a range of possible risk outcomes from the analysis.

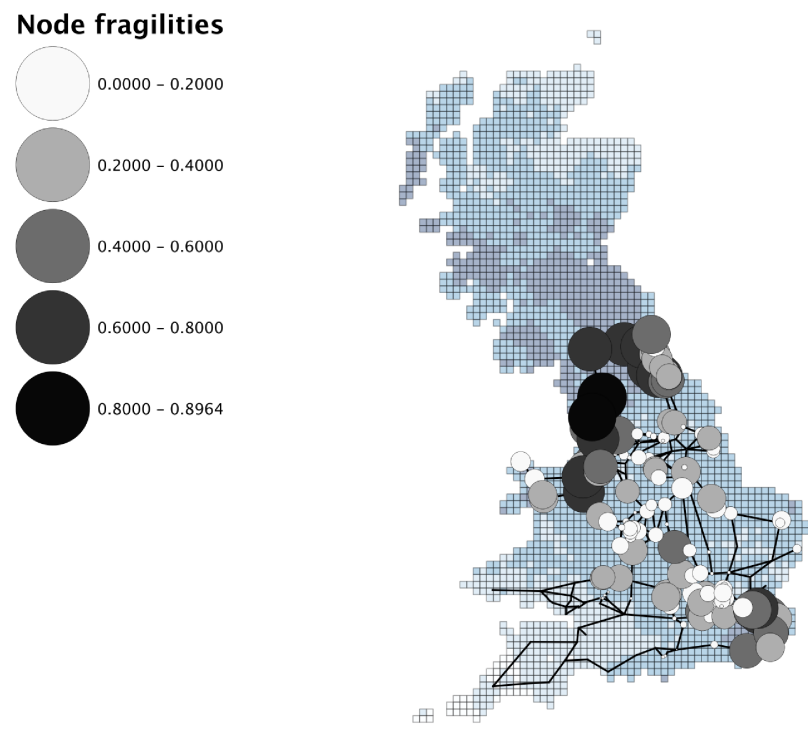

(a)

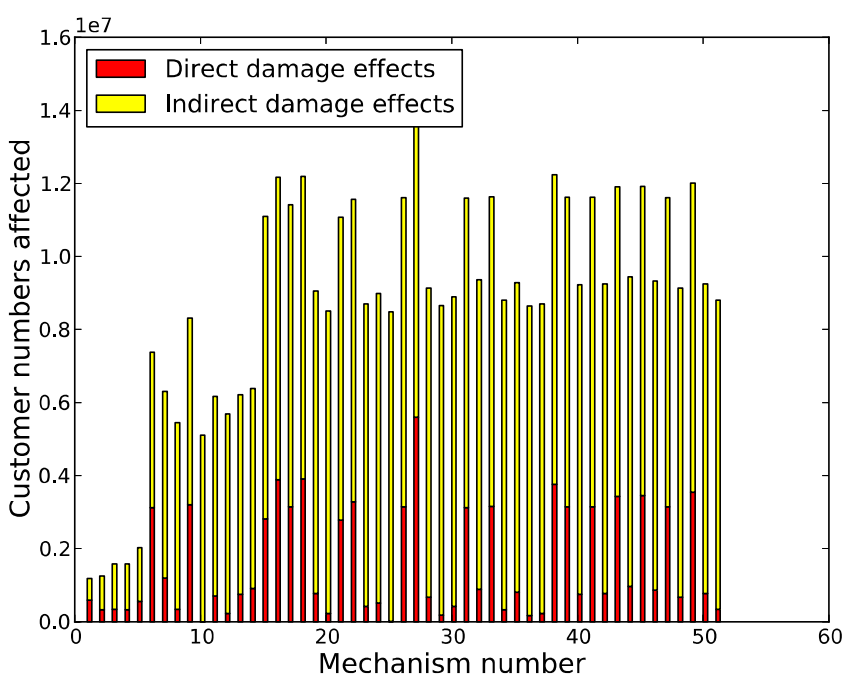

(b)

Figure 3. Figures showing (a) The network node fragilities (color coded and weighted by magnitude) for a particular hazard event, (b) Sample failure mechanisms and direct and indirect customers affected.

Another outcome of the analysis is shown in Figure 4 where the ranges of risks (in $£$ millions) are calculated for multiple mechanisms across different hazard events (given by their exceedance probabilities). This result is obtained by executing the components $\mathrm{D}$ and $\mathrm{F}$ in the Figure 2 framework. Figure 4 captures the uncertainty of the risk analysis across a range of different infrastructure provisions.

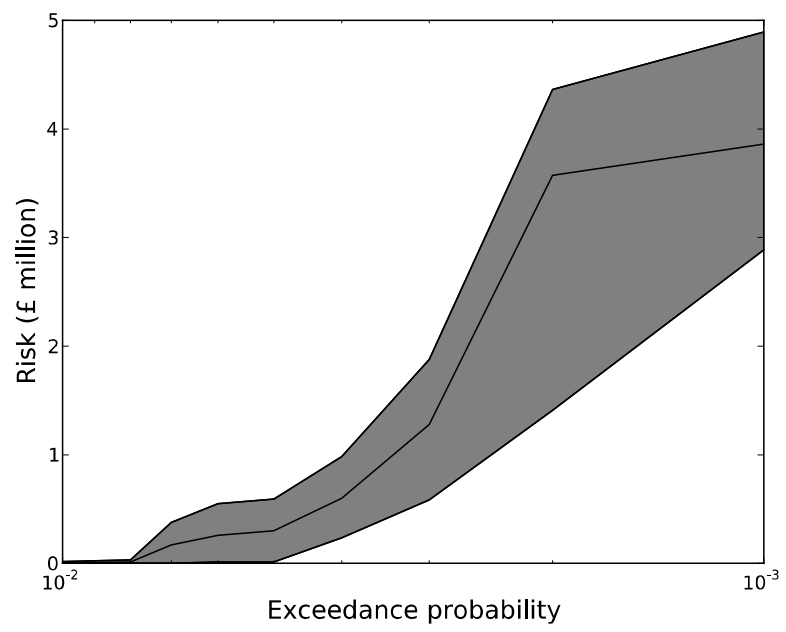

Figure 4. Plot of risks in $£$ million vs. hazard exceedance probabilities. The solid line shows the mean risk and the shaded boundaries show the maximum and minimum risk outcomes. 
International Symposium for Next Generation Infrastructure

October 1-4, 2013, Wollongong, Australia

\section{Acknowledgements}

The research reported in this paper was part of the UK Infrastructure Transitions Research Consortium (ITRC) funded by the Engineering and Physical Sciences Research Council under Programme Grant EP/I01344X/1.

\section{References}

${ }^{1}$ AECOM, Toward a City of Melbourne climate change adaptation strategy. A risk assessment and action plan discussion paper. Responding with resilience, Melbourne: AECOM, 2008.

${ }^{2}$ HM Treasury, National Infrastructure Plan 2011, The Stationary Office, London, 2011.

${ }^{3}$ Rinaldi, S. M., Peerenboom, J. P., and Kelly, T. K., "Identifying, understanding, and analyzing critical infrastructure interdependencies", Control Systems, IEEE, vol. 21, No. 6, 2001, pp. 11-25. http://dx.doi.org/10.1109/37.969131

${ }^{4}$ Little, R. G., "Controlling cascading failure: understanding the vulnerabilities of interconnected infrastructures", Journal of Urban Technology, Vol. 9, No. 1, 2002, pp. 109-123. http://dx.doi.org/10.1080/106307302317379855

${ }^{5}$ Baade, R. A., Baumann, R., and Matheson, V., "Estimating the economic impact of natural and social disasters, with an application to Hurricane Katrina", Urban Studies, Vol. 44, No. 11, 2007, pp. 2061-2076. http://dx.doi.org/10.1080/00420980701518917

${ }^{6}$ Pitt, M., Learning lessons from the 2007 floods, London: Cabinet Office, 2008.

${ }^{7}$ McEvoy, D., Ahmed, I., and Mullett, J., "The impact of the 2009 heat wave on Melbourne's critical infrastructure", Local Environment, Vol. 17, No. 8, 2012, pp. 783-796. http://dx.doi.org/10.1080/13549839.2012.678320

${ }^{8}$ ATSE, Assessment of impacts of climate change on Australia's physical infrastructure, Parkville, Victoria: Australian Academy of Technological Sciences and Engineering (ATSE), 2008.

${ }^{9}$ DEFRA, UK climate change risk assessment 2012: Evidence report, Department for Environment, Food \& Rural Affairs, The Stationary Office, London, 2012.

${ }^{10}$ Hall, J. W., Dawson, R. J., Sayers, P. B., Rosu, C., Chatterton, J. B., and Deakin, R., "A methodology for national-scale flood risk assessment", Proceedings of the ICE-Water and Maritime Engineering Conference, Vol. 156, No. 3, 2003, pp. 235-247.

${ }^{11}$ Bristow, M., Fang, L., \& Hipel, K. W., "System of Systems Engineering and Risk Management of Extreme Events: Concepts and Case Study", Risk Analysis, Vol. 32, No. 11, 2012, pp. 1935-1955. http://dx.doi.org/10.1111/j.1539-6924.2012.01867.x

${ }^{12}$ Pederson, P., Dudenhoeffer, D., Hartley, S., and Permann, M., Critical infrastructure interdependency modeling: a survey of US and international research, Idaho National Laboratory, 2006, pp. 1-20.

${ }^{13}$ Duenas-Osorio, L., and Vemuru, S. M., "Cascading failures in complex infrastructure systems”, Structural safety, Vol. 31, No. 2, 2009, pp. 157-167.

http://dx.doi.org/10.1016/j.strusafe.2008.06.007

${ }^{14}$ Okuyama, Y., "Modeling spatial economic impacts of an earthquake: Input-output approaches", Disaster Prevention and Management, Vol. 13, No. 4, 2004, pp. 297-306. http://dx.doi.org/10.1108/09653560410556519

${ }^{15}$ National Grid, Electricity Ten Year Statement, UK Electricity Transmission, National Grid, UK, 2012. 
International Symposium for Next Generation Infrastructure October 1-4, 2013, Wollongong, Australia

${ }^{16}$ Walford, N. S., and Hayles, K. N., "Thirty Years of Geographical (In) consistency in the British Population Census: Steps towards the Harmonisation of Small-Area Census Geography”, Population, Space and Place, Vol. 18, No. 3, 2012, pp. 295-313.

http://dx.doi.org/10.1002/psp.658 